TRANSACTIONS OF THE

AMERICAN MATHEMATICAL SOCIETY

Volume 358, Number 11, November 2006, Pages 4809-4820

S 0002-9947(06)03819-0

Article electronically published on January 24, 2006

\title{
ON $C^{\infty}$ AND GEVREY REGULARITY OF SUBLAPLACIANS
}

\author{
A. ALEXANDROU HIMONAS AND GERSON PETRONILHO
}

\begin{abstract}
In this paper we consider zero order perturbations of a class of sublaplacians on the two-dimensional torus and give sufficient conditions for global $C^{\infty}$ regularity to persist. In the case of analytic coefficients, we prove Gevrey regularity for a general class of sublaplacians when the finite type condition holds.
\end{abstract}

\section{InTRODUCTION AND RESULTS}

The problem of hypoellipticity (analytic, Gevrey, and $C^{\infty}$, local and global) for sublaplacians, although it has been studied extensively by many authors, is still the source of many unresolved questions, since there is no necessary and sufficient condition that characterizes hypoellipticity of a sublaplacian in its general form. Here, we begin with the study of zero order perturbations of the model operator $-\partial_{t}^{2}-a^{2}(t) \partial_{x}^{2}$, which is well known to be globally $C^{\infty}$ hypoelliptic in $\mathbb{T}^{2}$ when $a$ is a real valued function in $C^{\infty}(\mathbb{T})$ and is not identically equal to zero (see [FO], [H1]). Then we continue with the study of Gevrey regularity of a larger class of operators when the finite type condition holds.

For stating our results we need the following definitions. Let $s \geq 1$. We say that a function $f \in C^{\infty}\left(\mathbb{T}^{N}\right)$ is in the Gevrey class $G^{s}\left(\mathbb{T}^{N}\right)$ if there exists a constant $C>0$ such that $\left|\partial_{x}^{\alpha} f(x)\right| \leq C^{|\alpha|+1}(\alpha !)^{s}$, for all $\alpha \in \mathbb{Z}_{+}^{N}, x \in \mathbb{T}^{N}$. In particular, $G^{1}\left(\mathbb{T}^{N}\right)$ is the space of all periodic analytic functions, denoted by $C^{\omega}\left(\mathbb{T}^{N}\right)$. We recall that a distribution $u \in D^{\prime}\left(\mathbb{T}^{N}\right)$ is in $G^{s}\left(\mathbb{T}^{N}\right)$ if and only if there exist constants $\epsilon>0$ and $C>0$ such that

$$
|\hat{u}(\xi)| \leq C e^{-\epsilon|\xi|^{\frac{1}{s}}}, \quad \xi \in \mathbb{Z}^{N}
$$

A linear partial differential operator $P$ defined on $\mathbb{T}^{N}$ with coefficients in $C^{\infty}\left(\mathbb{T}^{N}\right)$ is said to be globally $C^{\infty}$ hypoelliptic in $\mathbb{T}^{N}$ if for any $u \in D^{\prime}\left(\mathbb{T}^{N}\right)$ the condition $P u \in C^{\infty}\left(\mathbb{T}^{N}\right)$ implies that $u \in C^{\infty}\left(\mathbb{T}^{N}\right)$. If $P$ has coefficients in $C^{\omega}\left(\mathbb{T}^{N}\right)$, then it is said to be globally $G^{s}$ hypoelliptic in $\mathbb{T}^{N}$ if for any $u \in D^{\prime}\left(\mathbb{T}^{N}\right)$ the condition $P u \in G^{s}\left(\mathbb{T}^{N}\right)$ implies that $u \in G^{s}\left(\mathbb{T}^{N}\right)$. When $s=1$ we say that $P$ is globally analytic hypoelliptic in $\mathbb{T}^{N}$.

Next we state our first result.

Received by the editors July 29, 2003 and, in revised form, August 5, 2004.

2000 Mathematics Subject Classification. Primary 35H10, 35B20.

Key words and phrases. Gevrey hypoellipticity, microlocal analysis, perturbations.

The first author was partially supported by the NSF under grant number DMS-9970857, and the second author was partially supported by CNPq.

(C)2006 American Mathematical Society Reverts to public domain 28 years from publication 
Theorem 1.1. Let $a$ and $c$ be real valued functions in $C^{\infty}(\mathbb{T})$, with $a \not \equiv 0$, and let $P$ be given by

$$
P=-\partial_{t}^{2}-a^{2}(t) \partial_{x}^{2}+c(t) .
$$

If there exist an open neighborhood $U \subset[-\pi, \pi)$ of the set $\left(a^{2}\right)^{-1}(0)$ and a constant $C$ such that

$$
c(t) \geq C a^{2}(t)-\frac{1}{8 \pi^{2}+\delta}, \delta>0, \forall t \in U,
$$

then $P$ is globally $C^{\infty}$ hypoelliptic in $\mathbb{T}^{2}$.

Remark 1.1. Two cases in which Theorem 1.1 applies are when $c(t) \geq-\frac{1}{8 \pi^{2}+\delta}$, or when $a^{2}$ and $c$ are linearly dependent over $\mathbb{R}$.

Remark 1.2. Using the ideas in the proof of Theorem 1.1 one may generalize it to include operators of the form

$$
P=-\partial_{t}^{2}-a^{2}(t) \partial_{x}^{2}+b(t) \partial_{x}+c(t)
$$

where $b \in C^{\infty}(\mathbb{T})$ and $a, c$ are as in Theorem 1.1. This generalization will be useful in proving Theorem 4.4 in Section 4.

If the finite type condition holds, which in the case of Theorem 1.1 reads $\partial_{t}^{k} a\left(t_{0}\right)$ $\neq 0$ for some nonnegative integer $k=k\left(t_{0}\right)$ for all $t_{0} \in \mathbb{T}$, then $P$ is locally hypoelliptic (see [Ho, $[\mathrm{RS}]$ ) and therefore globally hypoelliptic. However, when it fails and, in particular, when the zero set of $a(t)$ is large, then Theorem 1.1provides a sufficient condition for $P$ to be globally hypoelliptic, although it is not locally hypoelliptic. Condition (1.1) says that that $c(t)$ is not too negative on the zero set of $a(t)$. It is easy to see that if $a \equiv 0$ and $c=-m^{2}$, where $m$ is an integer, then $P$ is not globally hypoelliptic. Also, we recall that when $c \equiv 0$, then $P$ is globally hypoelliptic iff there is a $t_{0} \in \mathbb{T}$ such that $a\left(t_{0}\right) \neq 0$ (see [FO, [H1]). Is this true for any perturbation $c$ ? This is one of the questions we are exploring in this work. We mention here that one of the earliest results on zero order perturbations of sublaplacians is due to Stein $\underline{\underline{S}}$.

If $a$ is an analytic function in $\mathbb{T}$, then $P$ is globally analytic hypoelliptic in $\mathbb{T}^{2}$, for any $c \in C^{\omega}(\mathbb{T})$, thanks to Theorem 1.1 in $[\mathrm{CH}$. In our next theorem we shall extend this result into the Gevrey case.

Theorem 1.2. Let $P$ be given by

$$
P=-\sum_{j=1}^{\nu} X_{j}^{2}+X_{0}+c
$$

where

$$
X_{j}=\sum_{k=1}^{n} a_{j k}(t) \partial_{t_{k}}+\sum_{k=1}^{m} b_{j k}(t) \partial_{x_{k}}, j=0, \cdots, \nu,
$$

with $(t, x) \in \mathbb{T}^{n} \times \mathbb{T}^{m}$, and suppose that the coefficients $a_{j k}, b_{j k}$ are real-valued functions in $C^{w}\left(\mathbb{T}^{n}\right)$ and $c=c(t, x)$ is a real valued function in $C^{w}\left(\mathbb{T}^{n+m}\right)$. If the following conditions hold:

(i) every point of $\mathbb{T}^{n+m}$ is of finite type;

(ii) the vector fields $\sum_{k=1}^{n} a_{j k}(t) \partial_{t_{k}}, j=1, \cdots, \nu$, span $T_{t}\left(\mathbb{T}^{n}\right)$ for every $t \in \mathbb{T}^{n}$, then the operator $P$ is globally $G^{s}$ hypoelliptic in $\mathbb{T}^{n+m}$. 
We recall that a point in $\mathbb{T}^{n+m}$ is of finite type, if the Lie algebra generated by the vector fields $X_{0}, X_{1}, \cdots, X_{\nu}$ spans the tangent space of $\mathbb{T}^{n+m}$ there.

For results on the interesting open problems of local and global hypoellipticity of sublaplacians we refer the reader to the following works as well as the references listed therein: Amano [A], Baouendi and Goulaouic [BG], Bell and Mohammed [BM], Bove and Tartakoff [BT], Derridj D, Fedii [F], Gramchev, Popivanov and Yoshino GPY, Greenfield and Wallach GW, Himonas [H2, Himonas and Petronilho [H], Hörmander [Ho], Kohn [K], Oleinik and Radkevic $\mathrm{OR}$, Rodino $[\mathrm{R}$, Rothschild and Stein [RS], Taira [T], Tartakoff [Ta], and Treves [Tr].

\section{Proof of Theorem 1.1}

Let $u \in D^{\prime}\left(\mathbb{T}^{2}\right)$ such that

$$
P u=f, f \in C^{\infty}\left(\mathbb{T}^{2}\right) .
$$

By taking a partial Fourier transform in the last equation we obtain

$$
\left[-\partial_{t}^{2}+a^{2}(t) \xi^{2}+c(t)\right] \hat{u}(t, \xi)=\hat{f}(t, \xi)
$$

for all $\xi \in \mathbb{Z}$ and $t \in \mathbb{T}$. Since (2.1) is elliptic in $t$ we have that $\hat{u}(t, \xi) \in C^{\infty}(\mathbb{T})$ for each fixed $\xi$. Multiplying (2.1) by $\overline{\hat{u}}$ and integrating by parts with respect to $t \in \mathbb{T}$ gives

$$
\int_{\mathbb{T}}\left|\hat{u}_{t}(t, \xi)\right|^{2} d t+\int_{\mathbb{T}}\left[\xi^{2} a^{2}(t)+c(t)\right]|\hat{u}(t, \xi)|^{2} d t=\int_{\mathbb{T}} \hat{f}(t, \xi) \overline{\hat{u}}(t, \xi) d t .
$$

We shall need the following two lemmas.

Lemma 2.1. Under the hypotheses of Theorem 1.1 there exist constants $M_{1}>$ $0, L>0$ and an interval $I \subset \mathbb{T}$ such that

$$
\xi^{2} a^{2}(t)+d(t) \geq L, \quad \forall t \in I, \text { and }|\xi| \geq M_{1},
$$

where $d(t)=c(t)+\frac{1}{8 \pi^{2}+\delta}$.

Proof. If $t \in U$, then the hypothesis (1.1) implies that

$$
\xi^{2} a^{2}(t)+d(t) \geq \xi^{2} a^{2}(t)+C a^{2}(t)=a^{2}(t)\left(\xi^{2}+C\right)
$$

Since $U$ is an open neighborhood of $\left(a^{2}\right)^{-1}(0)$ there exists $t_{0} \in U \backslash\left(a^{2}\right)^{-1}(0)$. Thus $a^{2}\left(t_{0}\right)>0$. Hence there exist an interval $I \subset U$ and a constant $C_{1}>0$ such that

$$
a^{2}(t) \geq C_{1}, \quad \forall t \in I \text {. }
$$

It follows from (2.4) that

$$
\xi^{2} a^{2}(t)+d(t) \geq a^{2}(t)\left(\xi^{2}+C\right), \quad \forall t \in I .
$$

If $C \geq 0$, then for $t \in I$ it follows from (2.6) and (2.5) that

$$
\xi^{2} a^{2}(t)+d(t) \geq a^{2}(t)\left(\xi^{2}+C\right) \geq C_{1}\left(\xi^{2}+C\right) \geq C_{1}(1+C), \quad \forall|\xi| \geq 1 .
$$

Thus in the case $C \geq 0$ we have $L=C_{1}(1+C)$ and $M_{1}=1$. If $C<0$, then it follows from (2.6) and (2.5) that

$$
\xi^{2} a^{2}(t)+d(t) \geq a^{2}(t)\left(\xi^{2}+C\right) \geq C_{1}, \quad \forall t \in I, \text { and }|\xi| \geq \sqrt{1-C} .
$$

Hence in the case $C<0$ we have $L=C_{1}$ and $M_{1}=\sqrt{1-C}$. The proof of Lemma 2.1 is complete. 
Lemma 2.2. Under the hypotheses of Theorem 1.1 there exists a constant $M_{2}>0$ such that

$$
\xi^{2} a^{2}(t)+d(t) \geq 0, \quad \forall t \in \mathbb{T}, \text { and }|\xi| \geq M_{2},
$$

where $d(t)=c(t)+\frac{1}{8 \pi^{2}+\delta}$.

Proof. If $t \in U$, then the hypothesis (1.1) implies that

$$
\xi^{2} a^{2}(t)+d(t) \geq \xi^{2} a^{2}(t)+C a^{2}(t)=a^{2}(t)\left(\xi^{2}+C\right) .
$$

If $C \geq 0$, then it follows from (2.10) that

$$
\xi^{2} a^{2}(t)+d(t) \geq 0, \quad \forall t \in U, \xi \in \mathbb{Z} .
$$

If $C<0$, then it follows from (2.10) that

$$
\xi^{2} a^{2}(t)+d(t) \geq 0, \quad \forall t \in U, \quad|\xi| \geq \sqrt{-C} \doteq K_{1} .
$$

Let $W$ be an open neighborhood of the set $\left(a^{2}\right)^{-1}(0)$ such that $W \subset \bar{W} \subset U$. Thus the set $V \doteq[-\pi, \pi] \backslash W$ is a compact set. Also, let $m_{d}=\min _{t \in \mathbb{V}} d(t)$ and $m_{a}=\min _{t \in \mathbb{V}} a^{2}(t)$. Since $a^{2}(t)>0$ for all $t \in V$ we have $m_{a}>0$. Thus, for $t \in V$ we obtain

$$
\xi^{2} a^{2}(t)+d(t) \geq \xi^{2} a^{2}(t)+m_{d} \geq \xi^{2} m_{a}+m_{d}
$$

If $m_{d} \geq 0$, then it follows from (2.13) that

$$
\xi^{2} a^{2}(t)+d(t) \geq 0, \quad \forall t \in V, \xi \in \mathbb{Z} .
$$

If $m_{d}<0$, then it follows from (2.13) that

$$
\xi^{2} a^{2}(t)+d(t) \geq 0, \quad \forall t \in V,|\xi| \geq \sqrt{\frac{-m_{d}}{m_{a}}} \doteq K_{2} .
$$

We set $M_{2}=\max \left\{K_{1}, K_{2}\right\}$. If $C \geq 0$, then it follows from (2.11), (2.14) and (2.15) that

$$
\xi^{2} a^{2}(t)+d(t) \geq 0, \quad \forall t \in \mathbb{T},|\xi| \geq M_{2}
$$

which completes the proof in this case. If $C<0$, then it follows from (2.12), (2.14), and (2.15) that

$$
\xi^{2} a^{2}(t)+d(t) \geq 0, \quad \forall t \in \mathbb{T},|\xi| \geq M_{2}
$$

This completes the proof of Lemma 2.2

Going back to the proof of Theorem 1.1. we shall use the letter $C$ to represent a constant, which may change a finite number of times. For $|\xi| \geq M_{2}$ it follows from (2.2) and Lemma 2.2 that

$$
\begin{aligned}
\int_{\mathbb{T}}\left|\hat{u}_{t}(t, \xi)\right|^{2} d t & +\int_{\mathbb{T}}\left[\xi^{2} a^{2}(t)+d(t)\right]|\hat{u}(t, \xi)|^{2} d t=\int_{\mathbb{T}} \hat{f}(t, \xi) \overline{\hat{u}}(t, \xi) d t \\
& +\frac{1}{8 \pi^{2}+\delta} \int_{\mathbb{T}}|\hat{u}(t, \xi)|^{2} d t \\
& \leq\left.\left|\int_{\mathbb{T}} \hat{f}(t, \xi) \overline{\hat{u}}(t, \xi) d t+\frac{1}{8 \pi^{2}+\delta} \int_{\mathbb{T}}\right| \hat{u}(t, \xi)\right|^{2} d t \mid \\
& \leq\|\hat{f}(\cdot, \xi)\|_{L^{2}(\mathbb{T})}\|\hat{u}(\cdot, \xi)\|_{L^{2}(\mathbb{T})}+\frac{1}{8 \pi^{2}+\delta}\|\hat{u}(\cdot, \xi)\|_{L^{2}(\mathbb{T})} .
\end{aligned}
$$


By using the fundamental theorem of calculus we obtain

$$
|\hat{u}(t, \xi)|^{2} \leq 2\left(|\hat{u}(r, \xi)|^{2}+4 \pi\left\|\hat{u}_{t}(\cdot, \xi)\right\|_{L^{2}(\mathbb{T})}^{2}\right)
$$

$\forall t \in[-\pi, \pi], \forall r \in I$ and $\forall \xi \in \mathbb{Z}$. Moreover, since $P$ is elliptic in $I \times \mathbb{T}$, given $N \in \mathbb{N}$ there exists $C>0$ such that

$$
|\hat{u}(r, \xi)| \leq C|\xi|^{-2 N}, \forall r \in I, \quad \forall \xi \in \mathbb{Z} .
$$

Then, using (2.17) and (2.18) it follows that

$$
|\hat{u}(t, \xi)|^{2} \leq C|\xi|^{-2 N}+4 \pi\left\|\hat{u}_{t}(\cdot, \xi)\right\|_{L^{2}(\mathbb{T})}^{2}, \quad \forall t \in[-\pi, \pi] \text { and } \forall \xi \in \mathbb{Z} .
$$

Therefore, we have

$$
\int_{\mathbb{T}}|\hat{u}(t, \xi)|^{2} d t \leq C|\xi|^{-2 N}+\left.8 \pi^{2}|| \hat{u}_{t}(\cdot, \xi)\right|_{L^{2}(\mathbb{T})} ^{2} .
$$

By using (2.16) and (2.20) it follows that

$$
\begin{aligned}
\left\|\hat{u}_{t}(\cdot, \xi)\right\|^{2} & +\int_{\mathbb{T}}\left[\xi^{2} a^{2}(t)+d(t)\right]|\hat{u}(t, \xi)|^{2} d t \leq\|\hat{f}(\cdot, \xi)\|_{L^{2}(\mathbb{T})}\|\hat{u}(\cdot, \xi)\|_{L^{2}(\mathbb{T})} \\
& +C|\xi|^{-2 N}+a\left\|\hat{u}_{t}(\cdot, \xi)\right\|_{L^{2}(\mathbb{T})}, \quad \forall|\xi| \geq M_{2},
\end{aligned}
$$

where $a<1$. Thus it follows from (2.21) and Lemma 2.2 that for $|\xi| \geq M_{2}$ we have

$$
\begin{aligned}
& (1-a)\left(\left\|\hat{u}_{t}(\cdot, \xi)\right\|^{2}+\int_{\mathbb{T}}\left[\xi^{2} a^{2}(t)+d(t)\right]|\hat{u}(t, \xi)|^{2} d t\right) \\
\leq & \|\hat{f}(\cdot, \xi)\|_{L^{2}(\mathbb{T})}\|\hat{u}(\cdot, \xi)\|_{L^{2}(\mathbb{T})}+C|\xi|^{-2 N} .
\end{aligned}
$$

It follows from (2.17) that there exists a constant $C>0$ such that

$$
\|\hat{u}(\cdot, \xi)\|_{L^{2}(\mathbb{T})}^{2} \leq C\left(\left\|\hat{u}_{t}(\cdot, \xi)\right\|_{L^{2}(\mathbb{T})}^{2}+\int_{I}|\hat{u}(s, \xi)|^{2} d s\right) .
$$

For $|\xi| \geq K \doteq \max \left\{M_{1}, M_{2}\right\}$ it follows from (2.23), (2.3), and (2.9) that

$$
\begin{aligned}
\|\hat{u}(\cdot, \xi)\|_{L^{2}(\mathbb{T})}^{2} & \leq C\left(\left\|\hat{u}_{t}(\cdot, \xi)\right\|_{L^{2}(\mathbb{T})}^{2}+\int_{I} \frac{1}{L}\left(\xi^{2} a^{2}(s)+d(s)\right)|\hat{u}(s, \xi)|^{2} d s\right) \\
& \leq C\left(u_{t}(\cdot, \xi) \|_{L^{2}(\mathbb{T})}^{2}+\int_{\mathbb{T}}\left(\xi^{2} a^{2}(t)+d(t)\right)|\hat{u}(t, \xi)|^{2} d t\right) .
\end{aligned}
$$

Hence, for $|\xi| \geq K$,

$$
\begin{aligned}
\frac{1-a}{C}\|\hat{u}(\cdot, \xi)\|_{L^{2}(\mathbb{T})}^{2} & \leq(1-a)\left(\left\|\hat{u}_{t}(\cdot, \xi)\right\|_{L^{2}(\mathbb{T})}^{2}+\int_{\mathbb{T}}\left(\xi^{2} a^{2}(t)+d(t)\right)|\hat{u}(t, \xi)|^{2} d t\right) \\
(2.24) & \leq\|\hat{f}(\cdot, \xi)\|_{L^{2}(\mathbb{T})}\|\hat{u}(\cdot, \xi)\|_{L^{2}(\mathbb{T})}+C|\xi|^{-2 N}
\end{aligned}
$$

or

$$
\begin{aligned}
\|\hat{u}(\cdot, \xi)\|_{L^{2}(\mathbb{T})}^{2} & \leq C\|\hat{f}(\cdot, \xi)\|_{L^{2}(\mathbb{T})}\|\hat{u}(\cdot, \xi)\|_{L^{2}(\mathbb{T})}+C|\xi|^{-2 N} \\
& \leq C\|\hat{f}(\cdot, \xi)\|_{L^{2}(\mathbb{T})}^{2}+\frac{1}{2}\|\hat{u}(\cdot, \xi)\|_{L^{2}(\mathbb{T})}^{2}+C|\xi|^{-2 N} .
\end{aligned}
$$

For $|\xi| \geq K$ it follows from the last inequality that

$$
\|\hat{u}(\cdot, \xi)\|_{L^{2}(\mathbb{T})}^{2} \leq C|| \hat{f}(\cdot, \xi) \|_{L^{2}(\mathbb{T})}^{2}+C|\xi|^{-2 N} .
$$


Since $f \in C^{\infty}\left(\mathbb{T}^{2}\right)$, inequality (2.25) and the Cauchy-Schwarz inequality imply that given $N \in \mathbb{N}$ there exists $C>0$ such that

$$
|\hat{u}(\tau, \xi)| \leq C|\xi|^{-N},|\xi| \geq K .
$$

Since $P$ is elliptic at $\left(\tau_{0}, 0\right), \tau_{0} \in \mathbb{Z} \backslash\{0\}$, there exist constants $C>0$ and $L>0$ such that

$$
|\hat{u}(\tau, \xi)| \leq C(|\tau|+|\xi|)^{-N},|\tau|>L|\xi| .
$$

It follows from (2.26) that

$$
|\hat{u}(\tau, \xi)| \leq C(|\tau|+|\xi|)^{-N}, \quad|\tau|<\frac{3}{2} L|\xi| \text { and }|\xi| \geq K .
$$

Now, inequalities (2.27) and (2.28) imply that $u \in C^{\infty}\left(\mathbb{T}^{2}\right)$, since we have the estimate

$$
|\hat{u}(\tau, \xi)| \leq C(|\tau|+|\xi|)^{-N}, \quad(\tau, \xi) \in \mathbb{Z}^{2} \backslash F,
$$

where $F$ is a finite set of points in $\mathbb{Z}^{2}$. Therefore, the proof of Theorem 1.1 is complete.

\section{Proof of Theorem 1.2}

Let $u \in D^{\prime}\left(\mathbb{T}^{n+m}\right)$ such that

$$
P u=f \in G^{s}\left(\mathbb{T}^{n+m}\right) .
$$

It follows from hypothesis (i) that $u \in C^{\infty}\left(\mathbb{T}^{n+m}\right)$ thanks to Hörmander's Theorem (see $[\mathrm{Ho}]$ ). It follows from ([CH], Lemma 2.1) that there exists a positive constant $C$ such that

$$
\|v\|_{0} \leq C\left(\|P v\|_{0}+\|v\|_{-1}\right), \text { for } v \in C^{\infty}\left(\mathbb{T}^{n+m}\right) .
$$

Since $\partial_{x}^{\alpha} u \in C^{\infty}\left(\mathbb{T}^{n+m}\right)$, it follows from (3.2) that

$$
\begin{aligned}
\left\|\partial_{x}^{\alpha}\right\|_{0} & \leq C\left(\left\|P\left(\partial_{x}^{\alpha} u\right)\right\|_{0}+\left\|\partial_{x}^{\alpha} u\right\|_{-1}\right) \\
& \leq C\left(\left\|\partial_{x}^{\alpha}(P u)\right\|_{0}+\left\|\left[P, \partial_{x}^{\alpha}\right] u\right\|_{0}+\left\|\partial_{x}^{\alpha} u\right\|_{-1}\right) .
\end{aligned}
$$

The proof of the next result is slightly different from the one in $\mathrm{CH}$. However, for the sake of completeness we will include the proof.

Lemma 3.1. There exists a positive constant B such that

$$
\left\|\partial_{x}^{\alpha} u\right\|_{0} \leq B^{|\alpha|+1}(\alpha !)^{s}, \forall \alpha \in \mathbb{Z}_{+}^{m} .
$$

Proof. We will prove the lemma by induction on $\alpha$. For $\alpha=0$ it is easy to see that (3.4) holds true. We now suppose that (3.4) holds for $\beta<\alpha$.

Since $a$ and $f$ are in $G^{s}\left(\mathbb{T}^{n+m}\right)$, there exists a positive constant $A$ such that

$$
\left\|\partial_{x}^{\alpha} a\right\|_{0} \leq A^{|\alpha|+1}(\alpha !)^{s}, \forall \alpha \in \mathbb{Z}_{+}^{m}
$$

and

$$
\left\|\partial_{x}^{\alpha} f\right\|_{0} \leq A^{|\alpha|+1}(\alpha !)^{s}, \forall \alpha \in \mathbb{Z}_{+}^{m} .
$$

We recall that

$$
\left\|\partial_{x}^{\alpha} u\right\|_{-1} \leq\left\|\partial_{x}^{\alpha-e_{j}} u\right\|_{0},
$$

where $e_{j}$ is an element of the orthonormal basis of $\mathbb{R}^{m}$. 
Since the coefficients of $X_{j}, j=0, \cdots, \nu$, depend only on $t$, then the vector fields $X_{j}$ commute with $\partial_{x}^{\alpha}$ and we have

$$
\left[P, \partial_{x}^{\alpha}\right] u=a \partial_{x}^{\alpha} u-\partial_{x}^{\alpha}(a u)=-\sum_{\beta<\alpha}\left(\begin{array}{c}
\alpha \\
\beta
\end{array}\right) \partial_{x}^{\alpha-\beta} a \partial_{x}^{\beta} u .
$$

Therefore,

$$
\left\|\left[P, \partial_{x}^{\alpha}\right] u\right\|_{0} \leq \sum_{\beta<\alpha}\left(\begin{array}{c}
\alpha \\
\beta
\end{array}\right)\left\|\partial_{x}^{\alpha-\beta} a\right\|_{\infty}\left\|\partial_{x}^{\beta} u\right\|_{0} .
$$

Then by using (3.5), (3.6) and the induction hypothesis we obtain

$$
\left\|\left[P, \partial_{x}^{\alpha}\right] u\right\|_{0} \leq(\alpha !)^{s} \sum_{\beta<\alpha} A^{|\alpha|-|\beta|+1} B^{|\beta|+1} .
$$

By using (3.3), (3.6), (3.7) and (3.8) we obtain

$$
\left\|\partial_{x}^{\alpha} u\right\|_{0} \leq C\left(A^{|\alpha|+1}(\alpha !)^{s}+(\alpha !)^{s} \sum_{\beta<\alpha} A^{|\alpha|-|\beta|+1} B^{|\beta|+1}+B^{|\alpha|}\left(\alpha-e_{j}\right) !^{s}\right) .
$$

We look for $B$ of the form

$$
B=M A, \text { for some } M>1,
$$

such that (3.4) holds. By (3.9) it suffices to choose $M$ such that for all $\alpha \in \mathbb{Z}_{+}^{m}$ we have

$$
\begin{aligned}
& C\left(A^{|\alpha|+1}(\alpha !)^{s}+(\alpha !)^{s} \sum_{\beta<\alpha} A^{|\alpha|+2} M^{|\beta|+1}+A^{\alpha \mid} M^{|\alpha|}\left(\alpha-e_{j}\right) !^{s}\right) \\
\leq & A^{|\alpha|+1} M^{|\alpha|+1}(\alpha !)^{s} .
\end{aligned}
$$

By simplifying we obtain that the last inequality follows from

$$
C\left(1+A M \sum_{\beta<\alpha} M^{|\beta|}+\frac{1}{A} M^{|\alpha|}\right) \leq M^{|\alpha|+1}
$$

Since for $M>1$, we have

$$
\sum_{\beta<\alpha} M^{|\beta|} \leq\left[\left(\frac{M}{M-1}\right)^{m}-1\right] M^{|\alpha|}
$$

by (3.12) we see that for (3.11) to hold it suffices that

$$
C\left(\frac{1}{M^{|\alpha|+1}}+A\left[\left(\frac{M}{M-1}\right)^{m}-1\right]+\frac{1}{A M}\right) \leq 1 .
$$

Since the left-hand side of (3.13) goes to zero as $M$ goes to infinity, we conclude that there eixsts $M>1$ such that (3.13) holds and therefore (3.4) holds with $B=M A$. This completes the proof of Lemma 3.1 .

Recalling that

$$
\hat{u}(t, \xi)=\frac{1}{(2 \pi)^{m}} \int_{\mathbb{T}^{m}} e^{-i x \cdot \xi} u(t, x) d x
$$

it is easy to prove that

$$
\left|\xi^{\beta} \hat{u}(t, \xi)\right| \leq C|| \partial_{x}^{\beta} u \|_{0}, \quad \forall \beta \in \mathbb{Z}_{+}^{m} .
$$


It is standard to prove that (3.14) and (3.4) imply that there exist positive constants $C, \epsilon$ such that

$$
|\hat{u}(t, \xi)| \leq C e^{-\epsilon|\xi|^{\frac{1}{s}}}
$$

Since

$$
\hat{u}(\tau, \xi)=\frac{1}{(2 \pi)^{n}} \int_{\mathbb{T}^{n}} e^{-i t \cdot \tau} \hat{u}(t, \xi) d t,
$$

it follows thanks to (3.15) that

$$
|\hat{u}(\tau, \xi)| \leq C e^{-\epsilon|\xi|^{\frac{1}{s}}} .
$$

Now let $\left(t_{0}, x_{0}, \tau_{0}, \xi_{0}\right) \in T^{*}\left(\mathbb{T}^{n+m}\right) \backslash\{0\}$ be such that $\xi_{0} \neq 0$. Then there is $c>0$ such that

$$
\left(\tau_{0}, \xi_{0}\right) \in \Gamma \doteq\left\{(\tau, \xi) \in \mathbb{Z}^{n+m}:|\tau|<c|\xi|\right\} .
$$

Hence, by (3.16), we have

$$
|\hat{u}(\tau, \xi)| \leq C e^{-\epsilon^{\prime}|(\tau, \xi)|^{\frac{1}{s}}}, \forall(\tau, \xi) \in \Gamma .
$$

Inequality (3.17) together with similar microlocal estimates near each elliptic point $\left(t_{0}, x_{0}, \tau_{0}, 0\right), \tau_{0} \neq 0$ (see [Ho1, Theorem 8.6.1]), give the estimate

$$
|\hat{u}(\tau, \xi)| \leq C e^{-\epsilon^{\prime \prime}|(\tau, \xi)|^{\frac{1}{s}}}, \forall(\tau, \xi) \in \mathbb{Z}^{n+m},
$$

which shows that $u \in G^{s}\left(\mathbb{T}^{n+m}\right)$. The proof of Theorem 1.2 is complete.

\section{Further Results}

We would like to point out that condition (1.1) in Theorem 1.1 is not necessary for global $C^{\infty}$ hypoellipticity as the following result shows.

Theorem 4.1. Let $a$ and $c$ be real valued functions in $C^{\infty}(\mathbb{T})$. The operator $P=-\partial_{t}^{2}-a^{2}(t) \partial_{x}^{2}+c(t)$ is globally $C^{\infty}$ hypoelliptic in $\mathbb{T}^{2}$, if there is a $\delta$ such that $a(t) \neq 0$ for all $|t|>\delta$, and

$$
0<\delta \leq \sqrt{\frac{1}{64\left(\frac{1}{2}+\|c\|_{\infty}\right)}} .
$$

Example 4.1. Let $M$ be a large positive number and let $\delta$ be such that

$$
0<\delta \leq \sqrt{\frac{1}{64\left(\frac{1}{2}+M\right)}} .
$$

Choosing $a \in C^{\infty}(\mathbb{T})$ such that $a(t)=0$ for all $t \in\left(-\frac{\delta}{2}, \frac{\delta}{2}\right),|a(t)|>0$ for $|t|>\delta$, and $c(t)=-M$, we see that condition (1.1) in Theorem [1.1] is not true for the operator $P=-\partial_{t}^{2}-a^{2}(t) \partial_{x}^{2}-M$. However, Theorem 4.1 applies and shows that $P$ is globally $C^{\infty}$ hypoelliptic in $\mathbb{T}^{2}$.

Next we shall prove Theorem 4.1 For this, we need the following proposition.

Proposition 4.2. Let $P$ be as in Theorem 4.1. Let $u \in D^{\prime}\left(\mathbb{T}^{2}\right)$ such that $P u=$ $f \in C^{\infty}((-r, r) \times \mathbb{T})$ with $r>2 \delta$. Then $u \in C^{\infty}((-2 \delta, 2 \delta) \times \mathbb{T})$. 
Proof. Let $u \in D^{\prime}\left(\mathbb{T}^{2}\right)$ such that

$$
P u=f, f \in C^{\infty}((-r, r) \times \mathbb{T}), \text { where } r>2 \delta .
$$

We start by taking a partial Fourier transform in $P u=f$. Then we obtain the equation

$$
\left[-\partial_{t}^{2}+a^{2}(t) \xi^{2}+c(t)\right] \hat{u}(t, \xi)=\hat{f}(t, \xi)
$$

for all $\xi \in \mathbb{Z}$ and $|t|<r$.

For any $\xi$ fixed $\hat{u}(t, \xi)$ is in $C^{\infty}((-r, r))$ since (4.3) is elliptic in $t$. If we multiply (4.3) by $\overline{\hat{u}}$ and integrate in $t \in(-2 \delta, 2 \delta)$, then we obtain

$$
-\int_{-2 \delta}^{2 \delta} \hat{u}_{t t}(t, \xi) \overline{\hat{u}}(t, \xi) d t+\int_{-2 \delta}^{2 \delta}\left[\xi^{2} a^{2}(t)+c(t)\right]|\hat{u}(t, \xi)|^{2} d t=\int_{-2 \delta}^{2 \delta} \hat{f}(t, \xi) \overline{\hat{u}}(t, \xi) d t .
$$

If we integrate by parts the last equality, with respect to $t$, then we obtain

$$
\begin{aligned}
\int_{-2 \delta}^{2 \delta}\left|\hat{u}_{t}(t, \xi)\right|^{2} d t & -\left.\overline{\hat{u}}(t, \xi) \hat{u}_{t}(t, \xi)\right|_{t=-2 \delta} ^{t=2 \delta} \\
& +\int_{-2 \delta}^{2 \delta}\left[\xi^{2} a^{2}(t)+c(t)\right]|\hat{u}(t, \xi)|^{2} d t=\int_{-2 \delta}^{2 \delta} \hat{f}(t, \xi) \overline{\hat{u}}(t, \xi) d t .
\end{aligned}
$$

Then we use the Cauchy-Schwarz inequality to obtain

$$
\begin{aligned}
& \int_{-2 \delta}^{2 \delta}\left|\hat{u}_{t}(t, \xi)\right|^{2} d t+\int_{-2 \delta}^{2 \delta} a^{2}(t) \xi^{2}|\hat{u}(t, \xi)|^{2} d t \\
\leq & \int_{-2 \delta}^{2 \delta}\left[\frac{1}{2}+|c(t)|\right]|\hat{u}(t, \xi)|^{2} d t+\frac{1}{2} \int_{-2 \delta}^{2 \delta}|\hat{f}(t, \xi)|^{2} d t+\left|\overline{\hat{u}}(t, \xi) \hat{u}_{t}(t, \xi)\right|_{t=-2 \delta}^{t=2 \delta} \mid .
\end{aligned}
$$

Since $P$ is elliptic near $(\{-2 \delta\} \times \mathbb{T}) \cup(\{2 \delta\} \times \mathbb{T})$ and $P u=f \in C^{\infty}((-r, r) \times \mathbb{T})$, it follows that $u$ is $C^{\infty}$ near $(\{-2 \delta\} \times \mathbb{T}) \cup(\{2 \delta\} \times \mathbb{T})$. Thus, given $N \in \mathbb{N}$ there exists $C>0$ such that

$$
\begin{aligned}
& \int_{-2 \delta}^{2 \delta}\left|\hat{u}_{t}(t, \xi)\right|^{2} d t+\int_{-2 \delta}^{2 \delta} a^{2}(t) \xi^{2}|\hat{u}(t, \xi)|^{2} d t \\
\leq & \int_{-2 \delta}^{2 \delta}\left[\frac{1}{2}+\|c\|_{\infty}\right]|\hat{u}(t, \xi)|^{2} d t+C|\xi|^{-2 N}, \text { for all } \xi \in \mathbb{Z} \backslash\{0\} .
\end{aligned}
$$

If we write

$$
\hat{u}(t, \xi)=\hat{u}(-2 \delta, \xi)+\int_{-2 \delta}^{t} \hat{u}_{t}(s, \xi) d s,
$$

then it implies that given $N \in \mathbb{N}$ there exists $C>0$ such that

$$
\int_{-2 \delta}^{2 \delta}|\hat{u}(t, \xi)|^{2} d t \leq C|\xi|^{-2 N}+32 \delta^{2} \int_{-2 \delta}^{2 \delta}\left|\hat{u}_{t}(t, \xi)\right|^{2} d t .
$$

Since by hypothesis

$$
0<\delta \leq \sqrt{\frac{1}{64\left(\frac{1}{2}+\|c\|_{\infty}\right)}},
$$

then

$$
32 \delta^{2}\left(\frac{1}{2}+\|c\|_{\infty}\right)<\frac{1}{2} .
$$


By using (4.5), relation (4.6) gives

$$
\frac{1}{2} \int_{-2 \delta}^{2 \delta}\left|\hat{u}_{t}(t, \xi)\right|^{2} d t+\int_{-2 \delta}^{2 \delta} a^{2}(t) \xi^{2}|\hat{u}(t, \xi)|^{2} d t \leq C|\xi|^{-2 N} .
$$

For $|t|<2 \delta$ it is easy to prove that

$$
|\hat{u}(t, \xi)|^{2} \leq C\left(\int_{-2 \delta}^{2 \delta}\left|\hat{u}_{t}(t, \xi)\right|^{2} d t+\int_{-2 \delta}^{2 \delta} a^{2}(t)|\hat{u}(t, \xi)|^{2} d t\right) .
$$

It follows from (4.7) and (4.8) that

$$
|\hat{u}(t, \xi)| \leq C|\xi|^{-N}, \quad \forall \xi \in \mathbb{Z} \backslash\{0\},|t|<2 \delta .
$$

By using a standard microlocal analysis argument one can show that $u \in$ $C^{\infty}((-2 \delta, 2 \delta) \times \mathbb{T})$. The proof of Proposition 4.2 is complete.

Corollary 4.3. Let a be a real valued function in $C^{\infty}(\mathbb{T})$ such that $a(0)=0$ and $a(t) \neq 0, \forall t \in[-\pi, \pi] \backslash\{0\}$. Then $P=-\partial_{t}^{2}-a^{2}(t) \partial_{x}^{2}+c(t)$ is globally $C^{\infty}$ hypoelliptic in $\mathbb{T}^{2}$ for any real valued function $c \in C^{\infty}(\mathbb{T})$.

We conclude this work by considering a sublaplacian corresponding to a perturbation of the vector fields $\partial_{t}$ and $a(t) \partial_{x}$ in Theorem 1.1. Its hypoellipticity follows from Remark 1.2 and a concatenation formula.

Theorem 4.4. In $\mathbb{T}^{2}$ let the operator

$$
P=-\left(\partial_{t}+\phi(t)\right)^{2}-a^{2}(t)\left(\psi_{1}(t) \partial_{x}+\psi_{2}(t)\right)^{2},
$$

where the functions $\phi, \psi_{j}, j=1,2$, and $a \in C^{\infty}(\mathbb{T})$ are real valued. If $a \not \equiv 0$, $\psi_{1}(t) \neq 0, \forall t \in \mathbb{T}$, and $\int_{0}^{2 \pi} \phi(t) d t=0$, then $P$ is globally $C^{\infty}$ hypoelliptic in $\mathbb{T}^{2}$.

To prove Theorem 4.4 we shall need the following lemmas.

Lemma 4.5. Let the operator $L$ be given by

$$
L=-\partial_{t}^{2}-a^{2}(t)\left(\psi_{1}(t) \partial_{x}+\psi_{2}(t)\right)^{2},
$$

where $(t, x) \in \mathbb{T}^{2}$, and for $j=1,2$ the functions $\psi_{j}$ and $a \in C^{\infty}(\mathbb{T})$ are real valued. If $a \neq \equiv 0, \psi_{1}(t) \neq 0, \forall t \in \mathbb{T}$, then $P$ is globally $C^{\infty}$ hypoelliptic in $\mathbb{T}^{2}$.

Proof. This lemma follows from writing $L$ in the form

$$
L=-\partial_{t}^{2}-a^{2}(t) \psi_{1}^{2}(t) \partial_{x}^{2}-2 a^{2}(t) \psi_{1}(t) \psi_{2}(t) \partial_{x}-a^{2}(t) \psi_{2}^{2}(t),
$$

choosing $M=\max _{t \in \mathbb{T}}\left(\frac{\psi_{2}}{\psi_{1}}\right)^{2}(t)$, and observing that

$$
-a^{2}(t) \psi_{2}^{2}(t)=-\left(a \psi_{1}\right)^{2}(t)\left(\frac{\psi_{2}}{\psi_{1}}\right)^{2}(t) \geq-M\left(a \psi_{1}\right)^{2}(t) .
$$

Then $L$ is globally $C^{\infty}$ hypoelliptic, thanks to Remark 1.2.

Lemma 4.6. Let $L$ be as in Lemma 4.5 and $P$ as in Theorem 4.4. Then the concatenation

$$
L\left(e^{\int_{0}^{t} \phi(s) d s} u\right)=e^{\int_{0}^{t} \phi(s) d s} P u
$$

holds true for any $u \in D^{\prime}\left(\mathbb{T}^{2}\right)$. 
Proof. Let $u \in D^{\prime}\left(\mathbb{T}^{2}\right)$. Since $\phi \in C^{\infty}(\mathbb{T})$ and $\int_{0}^{2 \pi} \phi(t) d t=0$, we have

$$
e^{\int_{0}^{t} \phi(s) d s} \text { is in } C^{\infty}(\mathbb{T}) .
$$

We now consider the operator $L$ given in Lemma 4.5. Thus we have

$$
\begin{aligned}
L\left(e^{\int_{0}^{t} \phi(s) d s} u\right) & =-\partial_{t}^{2}\left(e^{\int_{0}^{t} \phi(s) d s} u\right) \\
& -a^{2}(t)\left(\psi_{1}(t) \partial_{x}+\psi_{2}(t)\right)^{2}\left(e^{\int_{0}^{t} \phi(s) d s} u\right) \\
& =e^{\int_{0}^{t} \phi(s) d s}\left[-\partial_{t}^{2} u-2 \phi(t) \partial_{t} u-\phi^{2}(t) u-\phi^{\prime}(t) u\right. \\
& \left.-a^{2}(t)\left(\psi_{1}(t) \partial_{x}+\psi_{2}(t)\right)^{2} u\right] \\
& =e^{\int_{0}^{t} \phi(s) d s}\left[-\left(\partial_{t}+\phi(t)\right)^{2} u\right. \\
& \left.-a^{2}(t)\left(\psi_{1}(t) \partial_{x}+\psi_{2}(t)\right)^{2} u\right] \\
& =e^{\int_{0}^{t} \phi(s) d s} P u .
\end{aligned}
$$

The proof of Theorem 4.4 follows from Lemmas 4.5 and 4.6 .

\section{REFERENCES}

[A] K. Amano, The global hypoellipticity of a class of degenerate elliptic-parabolic operators, Proc. Japan Acad. 60, Ser. 4, (1984), 312-314. MR0778515 (87f:35054)

[BG] M. S. Baouendi and C. Goulaouic, Nonanalytic-hypoellipticity for some degenerate elliptic operators, Bull. AMS 78, (1972), 483-486. MR0296507 (45:5567)

$[\mathrm{BM}]$ D. R. Bell and S. A. Mohammed, An extension of Hörmander's theorem for infinitely degenerate second-order operators, Duke Math J. 78, (1995), 453-475. MR1334203 (96g:35034)

[BT] B. E. Bove and D. Tartakoff, On a conjecture of Treves: Analytic hypoellipticity and Poisson strata, Indiana Univ. Math. J. 47 (1998), no. 2, 401-417. MR1647900 (2000d:35022)

$[\mathrm{CH}] \quad$ P. D. Cordaro and A. A. Himonas, Global analytic regularity for sums of squares of vector fields, Trans. Amer. Math. Soc. 350, (1998), 4993-5001. MR.1433115 (99b:35023)

[D] M. Derridj, Un probleme aux limites pour une classe d'operateurs du second ordre hypoelliptiques, Ann. Inst. Fourier, Grenoble 21, (1971), 99-148. MR0601055 (58:29139)

[F] V. S. Fedii, Estimates in $H^{s}$ norms and hypoellipticity, Dokl. Akad. Nauk SSSR 193 No. 2, (1970), 940-942. MR0271536(42:6419)

[FO] D. Fujiwara and H. Omori, An example of a globally hypoelliptic operator, Hokkaido Math. J. 12, (1983), 293-297. MR0719969 (86a:35038)

[GPY] T. Gramchev, P. Popivanov and M. Yoshino, Global properties in spaces of generalized functions on the torus for second order differential operators with variable coefficients, Rend. Sem. Mat. Pol. Torino 51 No.2, (1993), 145-172. MR1289385 (95k:35047)

[GW] S. J. Greenfield and N. R. Wallach, Global hypoellipticity and Liouville numbers, Proc. Amer. Math. Soc. 31, (1972), 112-114. MR0296508 (45:5568)

[H1] A. A. Himonas, On degenerate elliptic operators of infinite type, Math. Z. 220, no. 3, (1995), 449-460. MR1362255 (96j:35089)

[H2] A. A. Himonas, Global analytic and Gevrey hypoellipticity of sublaplacians under diophantine conditions, Proc. Amer. Math. Soc. 129, No. 7, (2000), 2061-2067. MR1825918 (2002c:35074)

[HP] A. A. Himonas and G. Petronilho, Global hypoellipticity and simultaneous approximability, J. Funct. Anal. 170 (2), (2000), 356-365. MR1740656 (2000m:35043)

[Ho] L. Hörmander, Hypoelliptic second order differential equations, Acta Math. 119, (1967), 147-171. MR0222474 (36:5526)

[Ho1] L. Hörmander, The Analysis of Linear Partial Differential Operators, Vol. I, SpringerVerlag, New York, (1983). MR0717035 (85g:35002a)

[K] J. J. Kohn, Pseudo-differential operators and hypoellipticity, Proc. Sympos. Pure Math. XXIII, (1973), 61-70. MR0338592(49:3356) 
[OR] O. A. Oleinik and E. V. Radkevic, Second order equations with nonnegative characteristic form, Amer. Math. Soc. and Plenum Press, (1973). MR0457908 (56:16112)

[R] L. Rodino, Linear Partial Differential operators in Gevrey spaces, World Scientific, (1993). MR.1249275 (95c:35001)

[RS] L. P. Rothschild and E.M. Stein, Hypoelliptic differential operators and nilpotent groups, Acta Math. 137, (1977), 247-320. MR0436223 (55:9171)

[S] E. M. Stein, An example on the Heisenberg group ralated to the Lewy operator, Invent. Math. 69, (1982), 209-216. MR0674401 (84c:35031)

[T] K. Taira, Le principe du maximum et l'hypoellipticite globale, Seminaire Bony-SöstrandMeyer, Expose no. I 348 No 7, (1984). MR0819767

[Ta] D. S. Tartakoff, Global (and local) analyticity for second order operators constructed from rigid vector fields on products of tori, Trans. AMS 348, no. 7, (1996), 2577-2583. MR 1344213 (96i:35018)

[Tr] F. Treves, Symplectic geometry and analytic hypo-ellipticity, La Pietra 1996 (Florence), 201-219, Proc. Sympos. Pure Math., 65, Amer. Math. Soc., Providence, RI, 1999. MR1662756 (2000b:35031)

Department of Mathematics, University of Notre Dame, Notre Dame, Indiana 46556

E-mail address: himonas.1@nd.edu

Department of Mathematics, Federal University of São Carlos, São Carlos, SP 13565-905, BRAZIL

E-mail address: gerson@dm.ufscar.br 